\title{
Compte rendu de Nickel. La naissance de l'industrie calédonienne, de Yann Bencivengo
}

Sarah Mohamed-Gaillard

\section{(2) OpenEdition \\ Journals}

Édition électronique

URL : http://journals.openedition.org/jso/7404

DOI : $10.4000 /$ jso. 7404

ISSN : $1760-7256$

Éditeur

Société des océanistes

\section{Édition imprimée}

Date de publication : 15 décembre 2015

Pagination : 360-362

ISBN : 978-2-85430-126-7

ISSN : 0300-953x

\section{Référence électronique}

Sarah Mohamed-Gaillard, " Compte rendu de Nickel. La naissance de l'industrie calédonienne, de Yann Bencivengo ", Journal de la Société des Océanistes [En ligne], 141 | juillet-décembre 2015, mis en ligne le 03 janvier 2016, consulté le 24 septembre 2020. URL : http://journals.openedition.org/jso/7404 ; DOI : https://doi.org/10.4000/jso.7404 
tard de sa vie, jusqu'à lui attirer les remontrances de Gordon, ne fut peut-être au départ qu'une façon de marquer à l'occidentale sa haute position sociale, mais ses besoins d'argent l'avaient fait recourir bien avant au vol ou au pillage de ses "sujets ", au bénéfice final des Blancs. Du reste, la cession de l'archipel à la couronne britannique pourrait être résumée à une histoire d'argent, ou plus exactement de " dette » à l'égard des Occidentaux. En 1858, ainsi que rapporte Seemann (1862: 246) plus d'une décennie avant sa signature,

"Bau was again in trouble. For various outrages asserted to have been committed against the life and property of American citizens, the Government of the United States demanded indemnity to Cakobau, as supreme chief of Bau and titular King of Fiji. The Corvette 'Vandalia, Captain Sinclair, has been sent to enforce the claim, and as the sum of 45,000 dollars was altogether beyond the means of the Fijian King to pay, overtures were made to $\mathrm{Mr}$. Pritchard for the cession of Fiji to Great Britain, on condition that this sum, which the natives were going to refund by assigning the proprietorship of 200,000 acres of land, be liquidated."

L'aura héroïque de Ma'afu, entré dans la légende de son vivant même, a une dimension idéologique dont l'historien peut tout au plus pointer ce qu'elle eut dès le départ de littéraire ou même de théâtral, citant en exergue de son travail Goethe et Euripide, auxquels on serait tenté d'ajouter Shakespeare : cette dimension reste sensible dans ce qu'en écrivent Fidjiens et Tongiens d'aujourd'hui. Mais la biographie de J. Spurway, qui constitue à elle seule une réussite et un exploit, ouvre surtout la voie à d'autres études sur ceux qui, acteurs, témoins ou victimes de la colonisation, les quelque 2000 Blancs établis dans l'archipel et les quelque 100000 autochtones, ont réellement fait que chaque parole de Ma'afu ne se réalise pas, autrement dit une véritable " histoire sociale » de la colonisation des îles Fidji.

\section{RÉFÉRENCES CITÉES}

Anonym, 1871 (May 6 $^{\text {th }}$ ). The Fiji Islands, The Pacific Commercial Advertiser Xv, 45, Honolulu.

CATHNEWS NEW ZEALAND, 2014 (August $\left.19^{\text {th }}\right)$. Fiji Catholics commemorate victory over Tongan invaders (http://cathnews. co.nz/2014/08/19/fiji-catholics-commemorate-victory-tongan-invaders/).

ERsKINE John Elphinstone, 1853. Journal of a Cruise among the Islands of the Western Pacific..., London, John Murray.

Gorran Hélène, 2011. Les rôles politiques des militaires fidjiens. Une histoire des guerriers, héros des conflits mondiaux, soldats de la paix, putschistes et hommes d'État, des premiers contacts avec les Occidentaux au gouvernement Bainimarama, thèse de l'université de la Nouvelle-Calédonie (http://portaildocumentaire.univ-nc.nc/files/public/bu/ theses_unc/TheseHeleneGoiran2011.pdf).

Greffrath Henry, 1871. Die politischen Zustände auf den Fidschi-Inseln, Zeitschrift des Gesellschaft für Erdkunde, Band 6, Berlin, pp. 540-548.

Lamy Étienne, 1902. Les missions catholiques françaises au XIXe siècle, Paris, Armand Colin.

LANGi Dave, 2010. What Would Have Happened, Had Maafu Toppled the Chiefs of Fiji, Matavuvale Network (http://www.matavuvale.com/group/yavusatonga/forum/topics/ what-would-have-happens-had).

Seemann Berthold, 1862. Viti. An Account of a Government Mission..., Cambridge, Macmillan.

Sundowner [Herbert Tichborne], 1896. Noqu Talaona. Stories from the South Seas, London, The European Mail.

—, 1897. Rambles in Polynesia, London, The European Mail.

Thomas Julian, 1886. Cannibals and Convicts. Notes of Personal Experiences in the Western Pacific, London, Cassell and Company.

Gilles Bounoure

Bencivengo Yann, 2014. Nickel. La naissance de l'industrie calédonienne, Tours, Presses universitaires François-Rabelais, coll. "Perspectives historiques ", , 420 p., glossaire, bibliogr., état des sources, chronologie, index, 89 illustrations noir et blanc et couleur, annexes comportant 106 illustrations en noir et blanc (photographies et cartes).

C'est dans la belle collection "Perspective historique" des Presses universitaires François Rabelais que Yann Bencivengo publie une monographie de La Société Le Nickel, de la découverte des gisements calédoniens par Jules Garnier en 1864

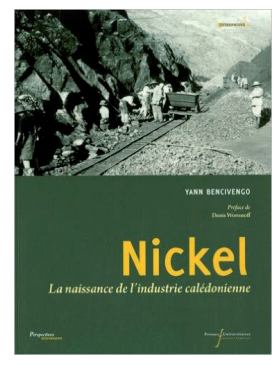
à la veille de la Première Guerre mondiale. Ce texte de 327 pages, suivi d'un volumineux dossier d'annexes, constitue une version remaniée de la thèse d'histoire que Yann Bencivengo a soutenue en 2010 à l'université Paris I et qui a reçu le prix Crédit agricole d'histoire des entreprises.

Partant de la découverte des gisements de nickel calédoniens qui bouleverse la donne de ce secteur économique, l'auteur s'attache à décrire les hommes qui, des dirigeants à la main d'œuvre, sont engagés dans le développement d'une industrie minière et métallurgique dans 
une colonie éloignée et quasiment dépourvue d'infrastructures. Yann Bencivengo, qui a eu accès aux archives de l'entreprise Le Nickel, a mobilisé de nombreux autres fonds publics et privés conservés à Paris, Aix-en-Provence, Roubaix et Nouméa. Cet ouvrage comble donc un manque historiographique important sur une entreprise et un secteur qui s'imposent comme des acteurs majeurs de la vie économique, politique et sociale de la Nouvelle-Calédonie.

Cette étude claire et rigoureuse explore en trois parties et neuf chapitres les différentes échelles de l'activité de la Société Le Nickel ; multinationale née à l'initiative d'acteurs calédoniens, construite sur les gisements de la Grande Terre, mais dont les activités dispersées de la Nouvelle-Calédonie à l'Europe sont marquées par le poids des distances tant géographiques que temporelles.

Yann Bencivengo analyse d'abord les bases de l'entreprise Le Nickel. Quelques années après sa découverte, le minerai calédonien, par sa teneur élevée en nickel, sa qualité et sa facilité d'extraction bouleverse les conditions du marché du nickel en dépit des coûts de transports élevés. Les débuts sont toutefois difficiles car il faut mettre au point le procédé technique permettant d'extraire le nickel du minerai, par ailleurs boycotté par les usiniers anglais et allemands qui cherchent à protéger de cette concurrence les mines européennes. Puis, la chute des cours du nickel entraîne la faillite de la banque de la NouvelleCalédonie. Au cours de ces premières années, John Higginson travaille au rapprochement des hommes et des entreprises investies dans le nickel calédonien, des fusions dont naît, en 1880, la société Le Nickel, qui s'appuie sur le procédé mis au point par Jules Garnier. Higginson, affairiste aventurier qui tend à pousser ses intérêts personnels en les présentant comme ceux de la colonie, accède ainsi à la direction de la succursale calédonienne de l'entreprise. En dépit d'une stratégie qui vise à étouffer les mineurs européens et à éviter le développement de toute concurrence en Nouvelle-Calédonie, la société Le Nickel est rapidement en difficulté et doit trouver de nouveaux partenaires industriels et commerciaux. Dans ces circonstances, la banque Rothschild, qui a consenti des avances à la société Le Nickel, entre dans son capital en 1883 avant d'en prendre le contrôle en 1885 , suite à l'éviction d'Higginson discrédité par des choix hasardeux.

Lauteur s'attarde ensuite sur la politique définie par la multinationale parisienne pour sa succursale calédonienne. Le Nickel poursuit la stratégie qui vise à évincer toute concurrence locale, tout en recherchant un mode de gestion lui permettant de surmonter les contraintes liées à l'éloignement entre le centre de décision parisien et le terrain calédonien. En 1889, décision est finalement prise de séparer géographiquement les directions et de leur assigner des compétences différentes : la direction parisienne est en charge des affaires commerciales et administrative, tandis que la direction de Nouméa est avant tout technique. L'heure est désormais celle des gestionnaires qui s'attachent à épurer les comptes de la société, à regagner la confiance des actionnaires et à étendre et rationnaliser le domaine minier de l'entreprise. Cette politique rigoureuse au service d'objectifs à long terme permet à la société Le Nickel d'être en position de force à la veille de la Grande guerre, d'autant que le nickel qui fut d'abord utilisé par l'industrie du luxe puis par les monnaies est désormais très demandé par l'industrie de l'armement. Parallèlement, la transformation locale du minerai reste une priorité partagée par le gouverneur Feillet qui fait du développement de l'économie de la colonie une priorité. Des dispositions sont ainsi prises pour favoriser la création d'usines métallurgiques, et c'est dans ce contexte qu'André Ballande construit sa fonderie de Doniambo, qui est opérationnelle en 1910.

Enfin, Yann Bencivengo analyse le fonctionnement de la succursale calédonienne, et tout d'abord la mise en œuvre des décisions prises par le siège parisien. La distance spatiale et temporelle de mềme que le manque d'infrastructures de la colonie constituent en effet de véritables contraintes, tant pour les communications que le transport du minerai. Il faut ainsi trois à quatre jours à cheval pour se rendre de Nouméa à Thio, le principal site minier du Nickel. De 1880 à 1885, le minerai extrait à Thio rejoint par cabotage la capitale de la colonie où il est transformé en mattes avant d'être expédié dans les usines du Havre, des environs de Birmingham et de Glasgow. A partir de 1890, une partie du minerai est expédiée depuis le port ouvert de Thio qui, avec l'ouverture de la fonderie de ThioMission, acquiert un rôle déterminant. Mais les rotations maritimes entre la Nouvelle-Calédonie et l'Europe sont longues - sept à huit mois de mer pour un aller-retour - irrégulières et périlleuses. Le passage des voiliers aux vapeurs en 1891 permet plus de rapidité, de régularité et de sécurité, mais 40 navires venus charger du minerai à Thio font néanmoins naufrage entre 1905 et 1907. La main d'œuvre constitue une autre préoccupation capitale de la succursale calédonienne. Les cadres et ingénieurs sont recrutés en France, mais il est difficile de trouver des candidats en raison de l'éloignement et de la mauvaise réputation de la colonie. Il faut aussi trouver pour les mines une force de travail suffisamment importante, stable et bon marché. Après le départ en 1883 des mineurs australiens qui ont participé à la découverte des gisements calédoniens, la société Le Nickel tente d'engager des colons-mineurs qui, en échange d'un total de 1500 jours de travail, obtiennent une maison et 5 hectares de terre. Après l'échec de cette tentative et concluant à l'inadaptation des travailleurs kanak aux travaux du front de 
taille, Le Nickel recourt à l'immigration néohébridaise. En 1882, 48 à 62\% des travailleurs de Thio ont ainsi été recrutés aux NouvellesHébrides. Mais, les conditions douteuses des recrutements poussent les autorités de la colonie à en suspendre la pratique de 1882 à 1884 , puis de 1885 à 1890. La société Le Nickel se tourne alors vers la population pénale de la Nouvelle-Calédonie et conclut, en 1887 et 1888 , quatre contrats avec l'administration pénitentiaire lui permettant d'employer des bagnards. Parallèlement, elle se tourne vers l'Asie. Le recrutement d'engagés sous contrat en 1884-1887 n'étant pas concluant, elle négocie avec le Japon, mais les 600 engagés qui arrivent dans la colonie en 1892 se plaignent vite de leurs conditions de travail et de vie. En 1900, l'administration pénitentiaire met un terme aux contrats qualifiés de chaire humaine et Le Nickel, privé de sa principale source de main d'œuvre, se dirige une nouvelle fois vers le Japon. Les travailleurs japonais, désormais recrutés sur le modèle des ouvriers européens, constituent $41 \%$ de la main-d'œuvre employée à Thio en 1900. Mais une fois encore, les relations se tendent entre ces travailleurs et Le Nickel. Notons enfin que la société recourt ponctuellement aux relégués et libérés, et que les engagés tonkinois et javanais sont peu employés par les mines avant 1914. Cette main-d'œuvre qui, entre 1889 et 1914 , oscille entre 700 à 1500 mineurs selon les pics de production, est soumise à des conditions de travail difficiles et dangereuses. Entre 1891 et 1895, 29 des 111 accidents qui se produisent à Thio sont mortels, et les éboulements en sont la principale cause. Il faut toutefois attendre 1915 pour que le gouverneur adopte un arrêté local sur les mesures destinées à assurer la sécurité du personnel. En dépit des difficultés liées à l'extraction du minerai et de sa transformation, les hommes qui s'impliquent dans ce secteur constituent un groupe assez stable. Ces hommes d'affaires et petits mineurs locaux sont à l'origine de nombreuses sociétés à durée de vie courte, en raison de leurs moyens financiers limités et de leurs lacunes techniques pour exploiter les concessions. Dans ce climat d'affairisme colonial, la Société Le Nickel et la maison Ballande à l'origine de la société des Hauts Fourneaux profitent des difficultés financières des petits mineurs et obtiennent de nouvelles concessions, ce qui leur permet d'étendre leur domaine minier et de s'imposer comme les leaders du secteur. En quelques années, la société Le Nickel devient donc le moteur de la vie économique de la NouvelleCalédonie et marque fortement et durablement l'archipel. Les flux de main d'œuvre qu'elle organise pour dégager la force de travail nécessaire à l'exploitation des mines, ont ainsi un impact sur l'évolution démographique de la NouvelleCalédonie. Le Nickel se voit rapidement reprocher de détourner la main d'œuvre pénale de la mise en valeur de l'archipel et de contribuer au maintien de la transportation qui entrave le développement de la colonisation libre. Sa position de quasi-monopole suscite aussi de nombreuses critiques, et Le Nickel fait peu à peu figure de véritable Etat dans l'Etat. Enfin, la constitution du domaine minier ne peut être déconnectés des spoliations foncières dont est alors victime la population kanak.

Signalons que l'ouvrage donne une large place aux illustrations. L'auteur a en effet porté un soin particulier à appuyer son analyse sur de nombreux graphiques, organigrammes, et tableaux et cartes qui éclairent particulièrement bien le texte. Le volume présente aussi une importante documentation iconographique - cartes postales et photographies commentées - qui donne de la chair au propos et constitue également une source. Le développement de 327 pages est en effet suivi d'un dossier de quelque quatrevingt pages, intitulé Études iconographiques et composé de huit sections : les fonderies de la société Le Nickel; le gisement Zizette; le plateau de Thio ; les plans inclinés du plateau de Thio, un exemple de système de descente des minerais vers la vallée; le transporteur des mines Bornet; les installations de la Société Le Nickel à Thio-Mission ; le centre de Poro. Un glossaire, une chronologie, un état des sources consultées, une bibliographique et un index achèvent cette étude fouillée.

Cette belle étude d'histoire économique constitue donc tout à la fois une monographie d'entreprise en contexte colonial, l'histoire d'une branche de la deuxième révolution industrielle et une histoire minière de la Nouvelle-Calédonie. Certes, le lecteur avant tout intéressé par la Nouvelle-Calédonie pourrait souhaiter de plus amples développements sur les relations de la société avec la population kanak dans la région de Thio, par exemple, ou sur l'influence politique que la société a pu exercer. Mais, il n'en reste pas moins que l'ouvrage et son auteur s'imposent comme des références pour une histoire de la mine en plein développement.

Sarah Mohamed-Gaillard, INALCO, CESSMA UMR 245

West-Sooby John (ed.), 2013. Discovery and Empire. The French in the South Seas, Adelaide, University of Adelaide Press, xiv-282 p., bibliogr., 18 ill.

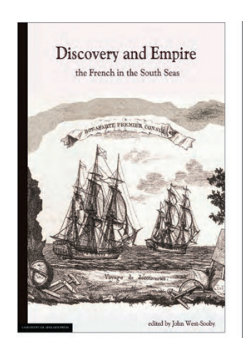

Ce volume, dont on peut consulter librement la version électronique sur le site de l'éditeur, a pour origine un colloque organisé en juillet 2009 dans le cadre de la XviI ${ }^{\text {th }}$ Biennial Conference of the Australasian Association of European Historians, alors 